\title{
Fine-Granularity Loading Schemes Using Adaptive Reed-Solomon Coding for xDSL-DMT Systems
}

\author{
Saswat Panigrahi and Tho Le-Ngoc \\ Department of Electrical and Computer Engineering, McGill University, 3480 University Street, Montréal, QC, Canada H3A $2 A 7$
}

Received 29 November 2004; Revised 9 May 2005; Accepted 22 July 2005

\begin{abstract}
While most existing loading algorithms for xDSL-DMT systems strive for the optimal energy distribution to maximize their rate, the amounts of bits loaded to subcarriers are constrained to be integers and the associated granularity losses can represent a significant percentage of the achievable data rate, especially in the presence of the peak-power constraint. To recover these losses, we propose a fine-granularity loading scheme using joint optimization of adaptive modulation and flexible coding parameters based on programmable Reed-Solomon (RS) codes and bit-error probability criterion. Illustrative examples of applications to VDSL-DMT systems indicate that the proposed scheme can offer a rate increase of about $20 \%$ in most cases as compared to various existing integer-bit-loading algorithms. This improvement is in good agreement with the theoretical estimates developed to quantify the granularity loss.
\end{abstract}

Copyright ( 2006 Hindawi Publishing Corporation. All rights reserved.

\section{INTRODUCTION}

Discrete multitone (DMT) modulation [1] has been widely used in xDSL applications such as asymmetric DSL (ADSL) [2] by the American National Standards Institute (ANSI) and the European Telecommunications Standard Institute (ETSI) and more recently for VDSL [3] by ANSI. Loading strategy is used for dynamic subcarrier rate and power allocation for given channel conditions, system constraints, and performance requirements.

For a multichannel total-power constrained problem, the optimal power distribution has long been known to be the "water-filling" distribution [4]. However the derivation tacitly assumes infinite granularity while most of the known modulation schemes support the integer number of bits per symbol. It was initially observed in $[5,6]$ that most of the granularity losses due to the integer number of bits per symbol could be recovered by rounding off rates to integers and scaling energies accordingly after starting with a water-filling [6] or flat on/off [5] energy distribution. However the freedom for such rescaling is considerably reduced in the presence of peak-power constraint.

Peak-power constraint $[7,8]$ arises from spectrum compatibility requirements to enable coexistence among multiple users and diverse services. When the peak-power constraint is far stricter than the total-power constraint, as is often the case in VDSL-DMT, there is hardly any room left for maneuverability (or rescaling) in the energy domain (to recover lapses in the bit-domain) and significant losses in achievable data rates of integer-bit algorithms are observed.

These losses accounting to be a significant percentage of the supported information rate compel us to tackle the integer-bit granularity problem through bit-error-rate-based joint optimization of adaptive modulation and flexible $\mathrm{RS}(n, k)$ coding on each subcarrier that can provide a wide range of fine choices in code rate and error-correction capability.

The remainder of the paper is organized as follows. Section 2 presents the overall optimization problem formulation and inferences from related literature about granularity. Section 3 develops a quantification of granularity loss based on relative strictness of peak-power and totalpower constraints. Section 4 describes the proposed adaptive Reed-Solomon-based fine-granularity loading (ARSFGL) scheme. Section 5 presents the illustrative results for various VDSL-DMT scenarios and concluding remarks are made in Section 6.

\section{POWER, INTEGER-BIT CONSTRAINTS, AND GRANULARITY LOSS}

Consider a xDSL-DMT system with $N$ subcarriers. Let $\varepsilon_{j}$ be the controllable transmitted power spectral density (PSD) and $\rho_{j}$ be the normalized channel signal-to-noise ratio when $\varepsilon_{j}=1$ over the $j$ th subcarrier, that is, $\rho_{j}$ is the ratio of the squared channel transfer function to the noise PSD over the 
$j$ th subcarrier. The noise includes both crosstalk and thermal additive white Gaussian noise (AWGN). The intercarrier spacing $\Delta f$ is assumed to be small enough for all the aforementioned PSDs to be nearly flat over each subcarrier.

The subcarrier rate function $b\left(\sigma_{j}\right)$ is defined as the maximum information rate in bits per symbol that can be supported at the received SNR of $\sigma_{j}=\rho_{j} \varepsilon_{j}$ to maintain the conceded error probability not exceeding a specified target value. The object function of the overall rate maximization problem is the total supported rate:

$$
R=\sum_{j=1}^{N} b\left(\rho_{j} \varepsilon_{j}\right)
$$

The traditional total-power constraint for the nontrivial power distribution can be expressed as

$$
\Delta f \cdot \sum_{j=1}^{N} \varepsilon_{j} \leq E_{\text {budget }} \quad \text { for } \varepsilon_{j} \geq 0,1 \leq j \leq N .
$$

In addition, many practical systems have limitation on the maximum transmit PSD. This implies the peak-power constraints:

$$
\varepsilon_{j} \leq \varepsilon_{j}^{\max }, \quad 1 \leq j \leq N,
$$

where $\left\{\varepsilon_{j}^{\max }\right\}_{j=1}^{N}$ is specified by the admissible transmit PSD mask, for example, SMClass3 in [8] or M1FTTCab in [3].

The subcarrier specific rate function can be expressed as

$$
b\left(\sigma_{j}\right)=r_{j}\left\lfloor\log _{2}\left(1+\frac{\sigma_{j}}{\Gamma_{j}}\right)\right\rfloor,
$$

where $r_{j}$ is the coding rate and $\Gamma_{j}$ is the SNR gap determined by the performance of the modulation and coding schemes in use. The floor operation (i.e., $\lfloor x\rfloor=m$ for the largest integer $m \leq x$ ) arises from the integer-bit constraint, since we try to find the largest integer number of bits per symbol that would satisfy the error rate target at SNR of $\sigma_{j}$. When the same FEC coding is applied for all subcarriers, that is, $r_{j}=r$, this floor operation restricts the subcarrier rate to have steps of $n r$ where $n$ is integer (i.e., integer-bit constraint) and $R=r \sum_{j=1}^{N}\left\lfloor\log _{2}\left(1+\left(\sigma_{j} / \Gamma_{j}\right)\right)\right\rfloor$.

Loading algorithms with objective to maximize rate (1) are called rate-adaptive (RA) loading algorithms. The totalpower only (TPO) constrained problem specified by (1) and (2) leads to the classical water-filling solution. Most RA algorithms $[5,6,9,10]$ addressed the TPO problem with integer-bit constraint. The more practical total and peakpower (TPP) constrained problem, that is, (1), (2), and (3), with integer-bit constraint was considered in $[5,7,11]$.

Both the greedy method in [9] and the Lagrangean method in [10] lead to the optimal solution for the integerbit TPO problem, the latter being much more computationally effective than the former. In $[5,6]$, a SNR-gap function[1] based method is proposed, which, in the initial stage, gives a continuous bit distribution resulting from a flat on/off and water-filling energy distribution, respectively. The difference between the rates resulting from these two energy distributions was seen to be only $2 \%$. To achieve negligible degradation due to the integer-bit constraint, both methods use bit-rounding and proper energy adjustment only for the TPO case.

In [5, Section 4.3.4], an Ad hoc extension for the TPP problem is presented by capping the bit round-off and the final energy rescaling with a maximal bit distribution and the peak energy constraint, respectively. A more formal treatment of the problem is presented in [7]. At first, the problem is solved without the integer-bit constraint for the general case of a continuously differentiable, strictly increasing, and strictly concave rate function. This solution is reproduced below with minor notational changes for easy reference,

$$
\begin{aligned}
& \text { if } \begin{aligned}
\Delta f \cdot \sum_{i=1}^{N} \varepsilon_{i}^{\max } & \leq E_{\text {budget }}, \quad \text { then } \varepsilon_{j}=\varepsilon_{j}^{\max } . \\
\text { If } \Delta f \cdot \sum_{i=1}^{N} \varepsilon_{i}^{\max } & >E_{\text {budget }}, \\
\text { then } \varepsilon_{j} & =\varepsilon_{j}(\lambda) \\
& =\varepsilon_{j}^{\max }, \quad \text { if } \lambda \leq \rho_{j} b_{\sigma}\left(\rho_{j} \varepsilon_{j}^{\max }\right) \\
& =\frac{1}{\rho_{j}} b_{\sigma}^{-1}\left(\frac{\lambda}{\rho_{j}}\right), \quad \text { if } \rho_{j} b_{\sigma}\left(\rho_{j} \varepsilon_{j}^{\max }\right) \\
& \leq \lambda \leq \rho_{j} b_{\sigma}(0) \\
& =0, \quad \text { if } \lambda \leq \rho_{j} b_{\sigma}(0),
\end{aligned}
\end{aligned}
$$

where $b_{\sigma}(\sigma)=\partial b(\sigma) / \partial \sigma$ and $b_{\sigma}^{-1}(\cdot)$ is the inverse of $b_{\sigma}(\cdot)$. The parameter $\lambda$ is the solution to

$$
\Delta f \cdot \sum_{j=1}^{N} \varepsilon_{j}(\lambda)=E_{\text {budget }}
$$

When (5) is satisfied, the energy distribution is independent of the rate function. Consequently, the peak-power constraint completely dominates and total-power constraint is trivially satisfied. In the rest of this paper, we will refer to this case as the peak-power only (PPO) case and by TPP we will mean the case when the inequality in (6) is satisfied, that is, both total-power and peak-power constraints play a role. A suboptimal algorithm for the TPP case with integer-bit constraint is presented in [7, Table IV]. The optimal algorithm (in terms of rate achieved) for the TPP case with integer-bit constraint is presented in [11].

The granularity loss in [7] is reported to be between 6$12 \%$ of the rate conveyed for the ADSL-TPP case, which is significant as compared to the variation of only $0.2-4 \%$ in the achievable rates of most existing integer-bit algorithms for the TPO case [12, Figure 4]. It is also higher than what would be expected from the $0.2 \mathrm{~dB}$ margin difference due to granularity reported for the ADSL-TPO case in $[5,13]$. This leads us to believe that granularity losses would grow with strictness in the peak-power constraint. Hence, we examine the case of VDSL-DMT for which the peak-power constraint is known to be particularly strict and also the number of subcarriers is large. 


\section{QUANTIFICATION OF GRANULARITY LOSS}

Let $\Omega \triangleq\left\{j \in\{1,2, \ldots, N\}: \varepsilon_{j}>0\right\}=\Omega_{1} \cup \Omega_{2}$ where $\Omega_{1} \cap \Omega_{2}=\varnothing, \Omega_{1} \triangleq\left\{j \in \Omega: b^{-1}\left(\left\lceil b\left(\rho_{j} \varepsilon_{j}\right)\right\rceil\right)>\rho_{j} \varepsilon_{j}^{\max }\right\}$, $\Omega_{2} \triangleq\left\{j \in \Omega: b^{-1}\left(\left\lceil b\left(\rho_{j} \varepsilon_{j}\right)\right\rceil\right) \leq \rho_{j} \varepsilon_{j}^{\max }\right\}$, and $\lceil x\rceil$ represents the ceiling operation (i.e., $\lceil x\rceil=n$ where $n$ is the smallest integer such that $x \leq n)$. It follows that $N_{\Omega}=N_{\Omega 1}+N_{\Omega 2}$ where $N_{\Omega}, N_{\Omega 1}$, and $N_{\Omega 2}$ are the cardinality of the sets $\Omega, \Omega_{1}$, and $\Omega_{2}$, respectively. $\Omega$ represents the set of nontrivially loaded subcarriers and $\Omega_{1}$ is the set of subcarriers in which ceiling the noninteger-bit $b\left(\rho_{j} \varepsilon_{j}\right)$ would cause the corresponding energy allocation to violate the peak-power constraint, that is, $\varepsilon_{j}>\varepsilon_{j}^{\max }$. Thus the only possibility to satisfy both the integer-bit and peak-power constraints in such a scenario is using the floor operation $\left\lfloor b\left(\sigma_{j}\right)\right\rfloor$. Hence the granularity loss for the $j$ th subcarrier is,

$$
\partial b_{j}^{G}=b\left(\sigma_{j}\right)-\left\lfloor b\left(\sigma_{j}\right)\right\rfloor, \quad \forall j \in \Omega_{1} .
$$

For subcarriers, where rounding is possible without violation of peak-power constraint:

$$
\partial b_{j}^{G}=b\left(\sigma_{j}\right)-\operatorname{round}\left(b\left(\sigma_{j}\right)\right), \quad \forall j \in \Omega_{2} .
$$

In both cases $\partial b_{j}^{G}$ can be treated as a quantization error with a quantization step of 1 . Since the variable to be quantized, $b\left(\sigma_{j}\right)$, has a much larger range (up to $15 \mathrm{bits} / \mathrm{symbol}$ ) than the quantization step, the granularity loss $\partial b_{j}^{G}$ can be considered as a uniformly distributed random variable (see [14, page 194]),

$$
\begin{gathered}
\partial b_{j}^{G} \sim U[0,1), \quad \forall j \in \Omega_{1} ; \\
\partial b_{j}^{G} \sim U\left[-\frac{1}{2}, \frac{1}{2}\right], \quad \forall j \in \Omega_{2} .
\end{gathered}
$$

The random variable representing the total granularity loss is $\partial b^{G}=\sum_{i \in \Omega} \partial b_{i}^{G}$ with its average

$$
\begin{aligned}
\overline{\partial b^{G}} & =E\left(\partial b^{G}\right)=\sum_{i \in \Omega_{1}} E\left(\partial b_{i}^{G}\right)+\sum_{i \in \Omega_{2}} E\left(\partial b_{i}^{G}\right) \\
& =N_{\Omega 1} \cdot \frac{1}{2}+N_{\Omega 2} \cdot 0=\frac{N_{\Omega 1}}{2} \\
& =\frac{\eta N_{\Omega}}{2}, \quad \text { where } 0 \leq \eta=\frac{N_{\Omega 1}}{N_{\Omega}} \leq 1,
\end{aligned}
$$

where $E(\cdot)$ in (11) represents the stochastic expectation operator. The ratio $\eta$ can be estimated as follows.

(i) TPO Case: In this case, by definition, there is no peakpower constraint or $\varepsilon_{j}^{\max }=\infty$; for all $j$, that is, $\Omega_{1}=\varnothing$ and $N_{\Omega 1}=0, \eta=0$. Also, due to the denominator being $\infty, E_{\text {budget }} / \Delta f \cdot \sum_{i=1}^{N} \varepsilon_{i}^{\max }=0$. Thus the average granularity loss is nearly zero, as observed in $[5,6,13]$.

(ii) PPO Case: In this case, $\varepsilon_{j}=\varepsilon_{j}^{\max }$; for all $j \in \Omega$, that is, $\Omega_{1}=\Omega$ and $\Omega_{2}=\varnothing$. Thus $N_{\Omega 1}=N_{\Omega}$ and $\overline{\partial b_{P P O}^{G}}=$ $N_{\Omega} / 2, \eta=1 . N_{\Omega}$ is fairly large in $\mathrm{xDSL}$ applications (e.g., more than 1000 in VDSL-DMT). Also from (5), $E_{\text {budget }} / \Delta f \cdot \sum_{i=1}^{N} \varepsilon_{i}^{\max } \leq 1$. (iii) TPP Case: For the TPP case, the analysis of $\eta$ is more involved and depends on the specific scenario. However, observing the values of $\eta$ in TPO and PPO cases, which act as the boundaries of the TPP case and its monotonic nature, we can consider the following approximation:

$$
\eta \approx\left\lceil\frac{E_{\text {budget }}}{\Delta f \cdot \sum_{i \in \Omega} \varepsilon_{i}^{\max }}\right\rceil^{1}, \quad\lceil x\rceil^{l} \triangleq \begin{cases}l, & x>l \\ x, & x \leq l .\end{cases}
$$

$\eta$ represents the relative strictness of the total-to-peak-power constraint and we can expect that as $\eta$ increases due to stricter peak-power constraint, granularity losses will be higher. It is worthwhile to note that for a general TPP case, as channel conditions worsen, $\Omega$ shrinks, thereby reducing the denominator of $\eta$. Eventually $\eta$ will increase to 1 and the TPP case will reduce to a PPO case and all previous inferences will apply. In VDSL-DMT application, $\eta$ is seen too fairly close to 1 in most cases and $N_{\Omega}$ is large, thus the granularity loss is expected to be a fairly significant percentage of the supported rate.

\section{ADAPTIVE REED-SOLOMON-BASED FINE-GRANULARITY LOADING SCHEME}

In the current $\mathrm{VDSL}^{1}$ system [3], as shown in Figure 1, there is only one fixed-rate $\operatorname{RS}(n, k)$ encoder with $n=255$ and $k=$ 239 in the PMS-TC layer and the bit and energy allocation are carried out only in the PMD layer.

The $\operatorname{RS}(255,239)$ coding is applied to bits that can be transmitted in various subcarriers. The coding channel is assumed to be a binary symmetric channel (BSC) with the crossover bit-error probability, $P_{e, \text { ch }}$ and $P_{e, \text { ch }}$ represents the BER averaged over all $N$ subcarrier DMT modems. The final system performance is represented by the post-decoding bit-error probability of the $\mathrm{RS}(n, k)$ code over $\mathrm{GF}\left(2^{m}\right)$ [15, Equations 4.23, 4.24]:

$$
\begin{gathered}
P_{e, \mathrm{dec}}\left(P_{e, \mathrm{ch}}, n, k\right) \leq \frac{2^{m-1}}{2^{m}-1} \sum_{i=t+1}^{n} \frac{i+t}{n}\left(\begin{array}{c}
n \\
i
\end{array}\right) P^{i}(1-P)^{n-i}, \\
\text { where } P=1-\left(1-P_{e, \mathrm{ch}}\right)^{m}, \quad t=\left\lfloor\frac{n-k}{2}\right\rfloor .
\end{gathered}
$$

The above upper bound is less than $0.1 \mathrm{~dB}$ away from the exact BER [16].

For RS $(255,239)$ with $m=8, n=255, k=239$, and $t=$ 8 , to achieve $P_{e, \mathrm{dec}} \leq 10^{-7}$, we need $P_{e, \mathrm{ch}}<10^{-3}\left(5.65 \times 10^{-4}\right.$ to be precise). This is ensured indirectly and approximately using the SNR gap method. Since only $M$-QAM is used, the uncoded SNR gap for $P_{e, \text { dec }} \leq 10^{-7}$ is nearly $9.75 \mathrm{~dB}$ for a large range of $M$ and also the $\operatorname{RS}(255,239)$ code is assumed to provide a uniform coding gain $\gamma_{c=} 3.75 \mathrm{~dB}$. Thus $\Gamma_{j}=\Gamma=$ $9.75-\gamma_{c}$ [17] and the code rate $r_{j}=r=239 / 255$ in (4).

\footnotetext{
${ }^{1}$ ADSL has a similar structure.
} 


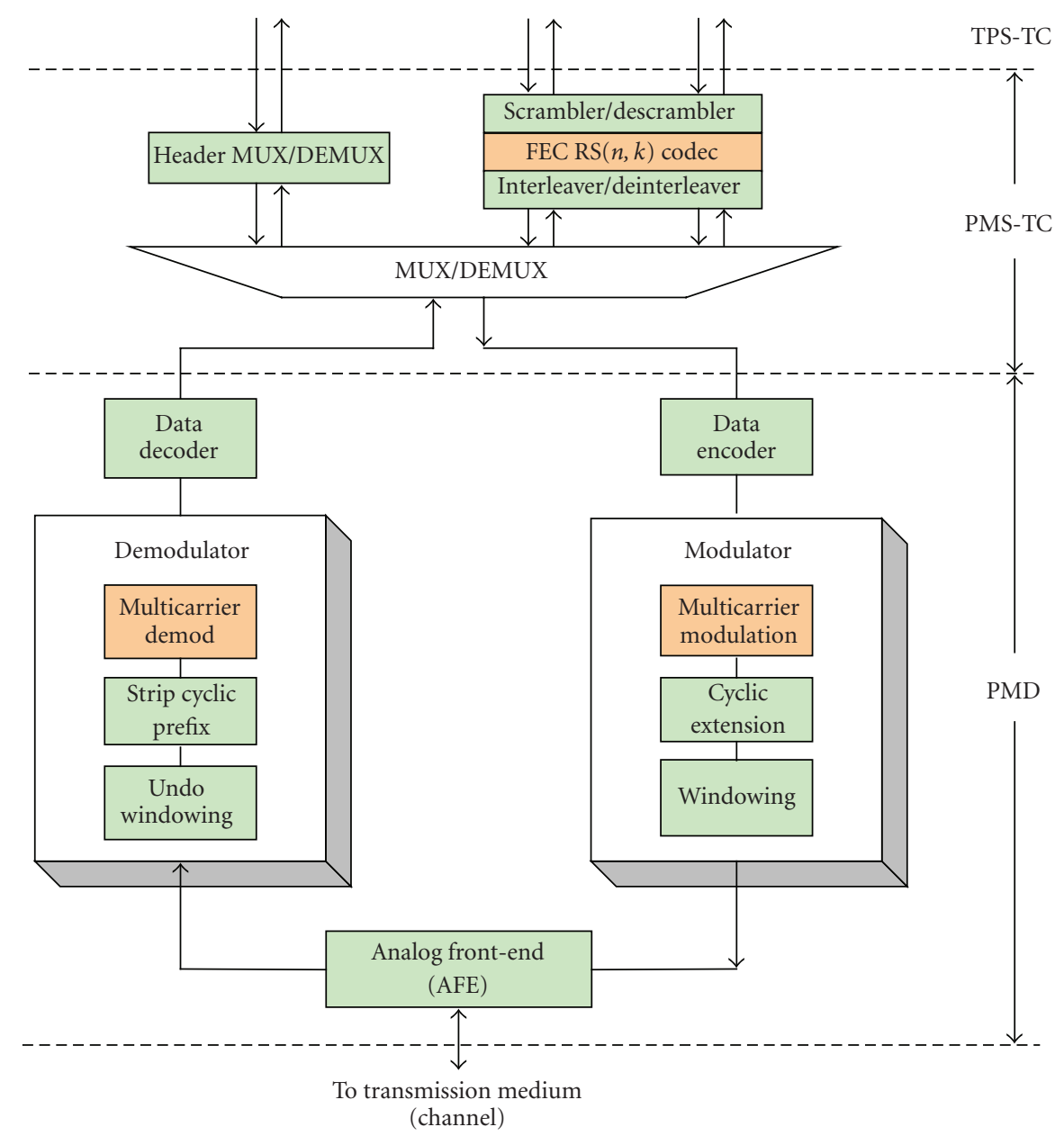

PMD: physical medium-dependant

PMS-TC: physical media specific transmission convergence

TPS-TC: transport protocol specific transmission convergence

FEC: forward error correction-RS $(255,239)$

FIgURE 1: Functional diagram of PMD and PMS-TC sublayers in current VDSL-DMT system.

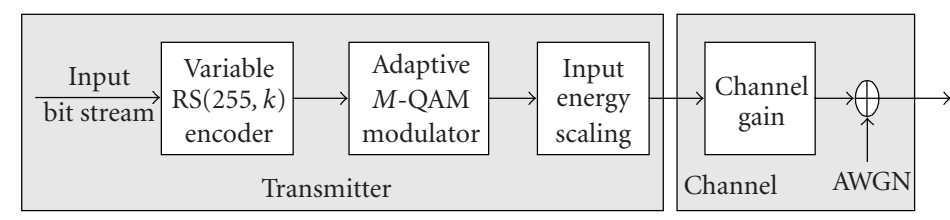

FIGURE 2: Subcarrier transmission model.

In the proposed adaptive RS-based fine-granularity loading (ARSFGL) scheme, instead of using a fixed-rate RS ( $n$, $k$ ) code for all subcarriers, we assume a variable rate RS ( $n$, $k_{i}$ ) code for each subcarrier $\# i$. This can be implemented by replacing the fixed-rate RS codec in Figure 1 with a single programmable $\operatorname{RS}(255, k)$ codec $[18,19]$, which operates on a per-subcarrier basis. Framing and buffering in MUX/DEMUX (Figure 1) will be modified accordingly to support this per-subcarrier RS codec operation and interleaving may not be required since independence of error patterns is maintained before decoding unlike in [3]. The loading algorithm provides the allocated rates (i.e., $k_{i}$, and the number of bits/symbol) and power as follows.

\subsection{Rate allocation}

Figure 2 depicts the equivalent model representing the transmission operation for each subcarrier. The complex symbol 
output of the M-QAM modulator is scaled to an input PSD level of $\varepsilon_{j}$ to achieve the overall received SNR, $\sigma_{j}=\varepsilon_{j} \rho_{j}$, corresponding to the $M_{j}$-QAM demodulator and $\operatorname{RS}\left(n, k_{j}\right)$ decoder bit-error probabilities of $P_{e, \mathrm{ch}}\left(M_{j}, \sigma_{j}\right)$ and $P_{e, \mathrm{dec}}\left[P_{e, \mathrm{ch}}\left(M_{j}, \sigma_{j}\right), n, k_{j}\right]$, respectively. Our optimization problem is formulated as follows:

$$
\begin{gathered}
\underset{k_{j}, M_{j}}{\operatorname{maximize}} b\left(\sigma_{j}\right)=\frac{k_{j}}{n} \times \log _{2} M_{j}, \\
\text { constraints : } k=1,3,5, \ldots, n, \\
\quad \log _{2} M_{j}=1,2,3, \ldots \\
P_{e, \mathrm{dec}}\left[P_{e, \mathrm{ch}}\left(M_{j}, \sigma_{j}\right), n, k_{j}\right] \leq 10^{-7} .
\end{gathered}
$$

$P_{e, \mathrm{dec}}\left[P_{e, \mathrm{ch}}\left(M_{j}, \sigma_{j}\right), n, k_{j}\right]$ is obtained from (13) with $k=$ $k_{j}$ and $P_{e, \mathrm{ch}}=P_{e, \mathrm{ch}}\left(M_{j}, \sigma_{j}\right)$.

$P_{e, \text { ch }}\left(M_{j}, \sigma_{j}\right)$ is the BER of $M_{j}$-QAM in AWGN channels, that is, for $\log _{2} M_{j}$ : odd with cross-QAM using impure Gray encoding [20],

$$
P_{e, \mathrm{ch}}(M, \sigma) \approx \frac{G_{p, M} N_{M}}{\log _{2} M} \cdot Q\left(\sqrt{\frac{2 \sigma}{C_{p, M}}}\right),
$$

where $G_{p, M}, N_{M}$, and $C_{p, M}$, represent the Gray penalty, number of nearest neighbors and packing coefficients, respectively. For validation purposes, we simulated crossconstellations constructed from the above scheme and we observe that (16) gives an accurate estimate of BER for all cross-constellations from $2^{5}, 2^{7}, \ldots, 2^{15}$ for BERs below 0.07 ,

For even $\log _{2} M_{j}$ with square-QAM using perfect Gray encoding [21],

$$
\text { for square-QAM [21]: } P_{e, \mathrm{ch}}(M, \sigma)=\frac{2}{\log _{2} M} \sum_{s=1}^{\log _{2} \sqrt{M}} P(s, \sigma) \text {, }
$$

where

$$
\begin{aligned}
P(s, \sigma)= & \frac{1}{\sqrt{M}} \\
& \times \sum_{i=1}^{\left(1-2^{-s}\right) \sqrt{M}-1}(-1)^{\left\lfloor i \cdot 2^{s-1} / \sqrt{M}\right\rfloor}\left[2^{s-1}-\left\lfloor\frac{2^{s-1} i}{\sqrt{M}}+\frac{1}{2}\right\rfloor\right] \\
& \times \operatorname{erf} c\left((2 i+1) \sqrt{\frac{3 \sigma}{2(M-1)}}\right) .
\end{aligned}
$$

Note that $b\left(\sigma_{j}\right)$ is a monotonously increasing with $k_{j}$ and $M_{j}, P_{e, \mathrm{ch}}\left(M_{j}, \sigma_{j}\right)$ and $P_{e, \mathrm{dec}}\left[P_{e, \mathrm{ch}}\left(M_{j}, \sigma_{j}\right), n, k_{j}\right]$ are monotonously increasing with $M_{j}$ and $k_{j}$, respectively. Thus we can search for $M_{j}$ and $k_{j}$ in a sequential manner. At first, $M_{j}$ is found to be within the limits specified by the uncoded case and the ideal Shannon limit, that is, $\left\lceil\log _{2}\left(1+\sigma_{j} / \Gamma\right)\right\rceil \leq$ $\log _{2} M_{j} \leq\left\lfloor\log _{2}\left(1+\sigma_{j}\right)\right\rfloor$. We then search for $k_{j}$ in descending order, that is, from $n$ to $(n-2),(n-5), \ldots$, until $P_{e, \text { dec }}\left[P_{e, \text { ch }}\left(M_{j}, \sigma_{j}\right), n, k_{j}\right] \leq 10^{-7}$. The optimum values for $k_{j}$ and $M_{j}$ for given $\sigma_{j}$ can also be precalculated and stored in a table such as Table 1 so that the search for $k_{j}$ and $M_{j}$ can be done by the table lookup technique.
TABLE 1: Example of rate lookup table.

\begin{tabular}{ccc}
\hline$\sigma(\mathrm{dB})$ & Optimum $k_{j}(1-255)$ & Optimum $\log _{2}\left(M_{j}\right)$ \\
\hline 30.0 & 245 & 8 \\
30.5 & 247 & 8 \\
31.0 & 249 & 8 \\
31.5 & 251 & 8 \\
32.0 & 229 & 9 \\
32.5 & 235 & 9 \\
33.0 & 239 & 9 \\
33.5 & 223 & 10 \\
34.0 & 229 & 10 \\
34.5 & 235 & 10 \\
35.0 & 239 & 10 \\
\hline
\end{tabular}

The optimized rate function (14) of the proposed ARSFGL is plotted along with that of the integer-bit-loading for VDSL in Figure 3. The finer granularity and inherent gains $^{2}$ in rate can be clearly seen. The gains stem from the fact that while $k$, and hence $P_{e, \mathrm{ch}}$, are fixed in the existing VDSL schemes, the proposed ARSFGL scheme varies $P_{e, \mathrm{ch}}\left(M_{j}, \sigma_{j}\right)$, jointly optimizing the adaptive coding and modulation schemes to achieve the maximum information rate. The gain in rate offered by the proposed ARSFGL is larger at higher SNR due to the fact that the proposed ARSFGL uses the bit-error probability (BER) criterion while the existing VDSL loading scheme is based on symbol-error probability (SER) [1]. As SNR increases, higher $M$ can be used and the difference between BER and SER becomes significant. Hence the BER-based ARSFGL is closer to the constraint $P_{e \text {, dec }} \leq 10^{-7}$. Another reason for choosing the BERbased scheme is that for the choice of $\operatorname{RS}\left(255, k_{j}\right)$ on each subcarrier, the input BER $P_{e, \mathrm{ch}}\left(M_{j}, \sigma_{j}\right)$ is a more meaningful quantity than the $M_{j}$-ary $\operatorname{SER}($ see (13)).

\subsection{Energy allocation}

As can be seen from Figure 3, the ARSFGL rate function is nondecreasing and can provide near-continuous rate adaptation. These conditions are sufficient for (5) to be satisfied ${ }^{3}$. Thus, for the PPO case, the optimal power allocation will be the PSD constraint. For the TPP case, however, the energy allocation depends on the rate function. Note that the solution for a continuously differentiable, strictly increasing, and strictly concave ratefunction is already available in (6) and (7). Furthermore, the ARSFGL rate function is close to the above properties. Therefore, we consider the rate function

\footnotetext{
2 Based on [3], no other code than RS is assumed in Figure 3. When additional or higher-performance coding is used, the gap between the Shannon limit and both curves in Figure 3 would be reduced by the same amount due to the additional coding gain. However, the granularity loss would remain the same.

${ }^{3}$ It is straightforward to verify (5) to hold for a continuous and increasing case. For the continuous and nondecreasing case, the only change is that (5) is no longer the unique optimum and solutions with smaller total energy might exist.
} 


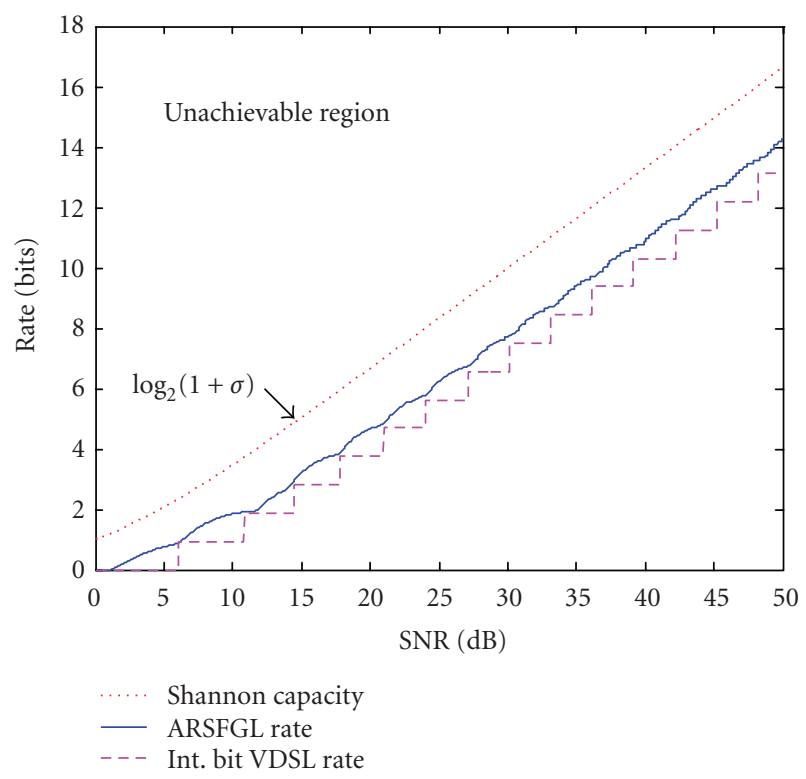

FIGURE 3: ARSFGL performance: rate versus SNR.

approximated by

$$
b_{e}(\sigma)=\alpha \log _{2}(\beta \sigma+\gamma)
$$

The approximation ${ }^{4}$ is achieved by curve-fitting and the values of $\alpha=0.9597, \beta=0.2736$, and $\gamma=0.8232$ yield a mean-squared error of less than 0.0076 bits.

From (6) and (7) with $b_{e}(\sigma)$ as the rate function, the final solution to the TPP energy allocation problem is

$$
\varepsilon_{j}=\left[\frac{B-\gamma}{\beta \rho_{j}}\right]_{0}^{\varepsilon_{j}^{\max }},
$$

where

$$
[x]_{l}^{m} \triangleq \begin{cases}m, & x \geq m \\ x, & 0<x<m \\ l, & x \leq l\end{cases}
$$

and $B$ is the solution to

$$
\Delta f \cdot \sum_{j=1}^{N}\left[\frac{B-\gamma}{\beta \rho_{j}}\right]_{0}^{\varepsilon_{j}^{\max }}=E_{\text {budget }}
$$

Here $B$ relates to $\lambda$ in (6) and (7) as $B=\alpha / \lambda \ln 2$. Thus the energy allocation problem reduces to the evaluation of $B$. This is done by using the low cost secant-based search

\footnotetext{
4 The approximation is done only for the purpose of energy allocation so that (5)-(7) can be directly used. However, the rate allocation following this energy allocation is done using the lookup table.
}

method proposed in [7] with the following minor changes to suit our notation and special usage of $(19), f(B)=\Delta f$. $\sum_{j=1}^{N}\left[B-\gamma / \beta \rho_{j}\right]_{0}^{\varepsilon_{j}^{\max }}-E_{\text {budget }}, b_{0} \equiv \min _{1 \leq i \leq N}\left\{\gamma / \beta \rho_{j}\right\}$, and $b_{1} \equiv \max _{1 \leq i \leq N}\left\{\varepsilon_{j}^{\max }+\gamma / \beta \rho_{j}\right\} . b_{0}$ and $b_{1}$ are the limits of the secant-based search. After incorporating these changes, the pseudocode in [7, Table I] can be directly used. It is worthwhile to note that by virtue of providing near-continuous rate adaptation, a secondary iterative procedure characteristic to integer-bit algorithms (e.g., bit-rounding and energyadjustment in $[5,6]$ or bisection search in [7]) is not necessary. Thus the energy allocation for ARSFGL is simpler.

\section{ILLUSTRATIVE EXAMPLES FOR APPLICATION TO VDSL-DMT SYSTEMS}

We consider the 4 transmit PSDs specified for VDSL-DMT [3, Section 7.1.2] in both upstream (US) and downstream (DS) and total-power budget over the same band [3, Table 7.1], to evaluate if the inequality in (5) holds and thereby classify the case as PPO or TPP. As shown in Table 2, all 5 shaded sections (including all US cases and the M1 FTTCab DS case) are under PPO constraint and the remaining 3 cases under TPP constraint. The TPO case does not occur in practice because admissible spectral masks for virtually every application have been specified $[3,8]$, but has been presented here for the sake of completeness.

\subsection{Evaluation of PPO case}

For PPO cases, (5) indicates that the energy allocation is independent of the rate allocation function. Thus all existing algorithms would result in the same solution because they 
TABLE 2: Occurrence of PPO and TPP cases in VDSL-DMT.

\begin{tabular}{l|cc|cc}
\hline \multirow{2}{*}{ PSD } & \multicolumn{2}{|c|}{ Upstream } & \multicolumn{2}{c}{ Downstream } \\
\cline { 2 - 5 } & $\Delta f \cdot \sum_{i} \varepsilon_{i}^{\max }(\mathrm{dBm})$ & $E_{\text {budget }}(\mathrm{dBm})$ & $\Delta f \cdot \sum_{i} \varepsilon_{i}^{\max }(\mathrm{dBm})$ & \multicolumn{1}{c}{$E_{\text {budget }}(\mathrm{dBm})$} \\
\hline M1 FTT Cab & 6.94 & 14.5 & 14.47 & 11.5 \\
M2 FTT Cab & 13.26 & 14.5 & 20.54 & 1.5 \\
M1 FTT Ex & 6.94 & 14.5 & 21.52 & 14.5 \\
M2 FTT Ex & 13.26 & 14.5 & 14.5 \\
\hline
\end{tabular}

TABLE 3: Simulation parameters.

\begin{tabular}{l|l|l}
\hline Number of subcarriers: & \multicolumn{4}{|l}{4096} \\
Cyclic prefix length: & 640 samples \\
US carriers: & U1: 870-1205, U2: 1972-2782 \\
DS carriers: & D1: 33-869, D2: 1206-1972 & \multicolumn{1}{|c}{ TPP } \\
\hline Loop and basic noise: & Loop 1 with AWGN $(-140 \mathrm{dBm} / \mathrm{Hz})+20$ VDSL xTalkers \\
\hline & PPO & Downstream \\
\hline Direction: & Upstream & $11.5 \mathrm{dBm}$ \\
Total-power constraint: & $14.5 \mathrm{dBm}$ & M2 FTTCab \\
Tx PSD constraint: & M1 FTTCab & + Alien noise F \\
Additional noise: & + Alien noise A &
\end{tabular}

strive for optimization in the energy domain and in this case the energy distribution is completely decided by the peakpower constraint. The received the SNR profile as a result of any loading algorithm would be $\sigma_{j}=\varepsilon_{j}^{\max } \rho_{j}$.

The general simulation parameters and those specific to the PPO case are presented in Table 3. This configuration resembles Test case-1 in [22] except that we do not fix the data rate at $10 \mathrm{Mbps}$, and study its variation over a wide range of loop lengths. The received SNR profile $\left\{\sigma_{i}\right\}_{i=1}^{N}$ and rate allocation over the subcarriers for this configuration at $2400 \mathrm{ft}$ are presented in Figures 4(a) and 4(b), respectively. The resulting data rates offered by the integer-bit-loading algorithm and proposed ARSFGL schemes are 10.94 Mbps and $13.41 \mathrm{Mbps}^{5}$, respectively. In other words, the proposed ARSFGL scheme provides an increase in rate of $22.6 \%$ (= 13.41/10.94-1). The rate-reach curves for different schemes are presented in Figure 4(c). Any integer-bit-loading algorithm would result in this same distribution as shown for the coded and uncoded cases. The proposed ARSFGL offers a much better reach-rate curve than the integer-bit-loading algorithm. The "theoretical expectation" curve is generated by adding $\overline{\partial b_{P P O}^{G}}$, that is, (11) with $\eta=1$, to the reachrate curve of the integer-bit-loading algorithm for the coded case at each reach value. The ARSFGL curve closely follows the "theoretical expectation" for distances longer than $1800 \mathrm{ft}$. However, for distances shorter than $1800 \mathrm{ft}$, it is noticeable that the ARSFGL curve is better due to the improvements arising from a BER-based loading. Shorter distances allow higher SNR and hence higher $M_{j}$. Therefore, the BER-based improvement is more pronounced as previously discussed (Figure 3 ). The improvements offered by

\footnotetext{
${ }^{5}$ It is worth noting that to achieve this increased rate with the integer-bitloading algorithm, a coding gain of $8.6 \mathrm{~dB}$ would be required, assuming 1 bit redundancy per-subcarrier characteristic of TTCM schemes [23].
}

the proposed ARSFGL are $23.6 \%, 27.5 \%$, and $70 \%$ at loop lengths of $2500 \mathrm{ft}, 3600 \mathrm{ft}$ and $4000 \mathrm{ft}$, respectively.

\subsection{Evaluation of TPP cases}

In TPP cases, the peak-power constraint is less stringent than the PPO case, and hence there is some room for maneuverability in the energy domain to recover some of the granularity losses.

The simulation parameters specific to the TPP case are presented in Table 3. This configuration resembles test case25 in [22] except that we do not fix the data rate at $22 \mathrm{Mbps}$, and study its variation over a wide range of loop lengths. The channel SNR $\rho_{j}$ for the above configuration and a loop length of $2100 \mathrm{ft}$ is shown in Figure 5(a). In Figure 5(b), the PSDconstraint in the form of M2FTTCab mask is presented along with the transmit PSD allocated by the ARSFGL scheme and integer-bit scheme by Baccarelli [7]. The integer-bit scheme leads to a sawtooth distribution, which deviates on both sides of the smooth distribution of the ARSFGL scheme. In Figure 5(c), the resulting bit distributions are presented. Unlike in the PPO case (where $\Omega_{2}=\varnothing$ ), here we observe sets of subcarriers (belonging to $\Omega_{2}$ ) where the integer-bit scheme is able to allocate more bits than the ARSFGL scheme due to the sawtooth nature of the energy distribution. This is what we have referred to as recovery of granularity loss through energy readjustment in earlier parts of the paper. It can be seen that, in the subcarrier 33-300 where the M2 mask is particularly stringent at $-60 \mathrm{dBm} / \mathrm{Hz}$, the ARSFGL scheme is always able to allocate more bits just like in PPO cases. These subcarriers form a part of set $\Omega_{1}$.

The rate-reach curves are presented in Figure 6(a). The rates achieved by the ARSFGL scheme for TPP case is compared with 3 integer-bit algorithms-Chow's TPP algorithm [5, Section 4.3.4], Baccarelli's (suboptimal) integer-bit 


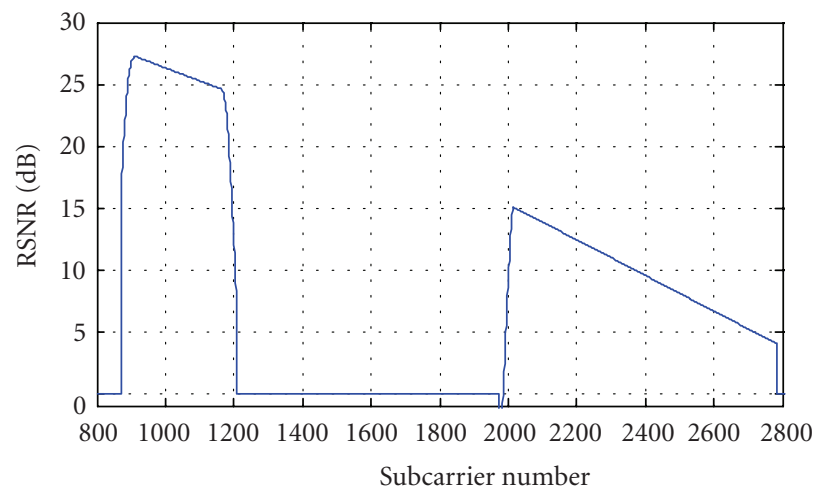

(a)

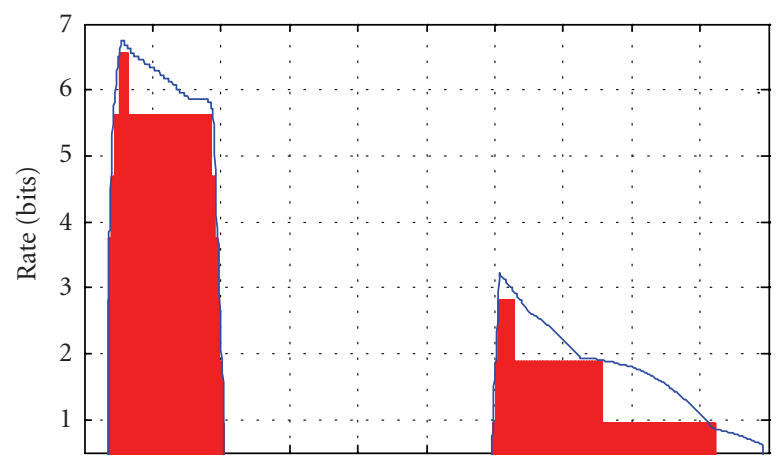

$800 \quad 1000 \quad 1200 \quad 1400160018002000 \quad 2200 \quad 2400 \quad 2600 \quad 2800$

Subcarrier number

- ARSFGL

Int. bit

(b)

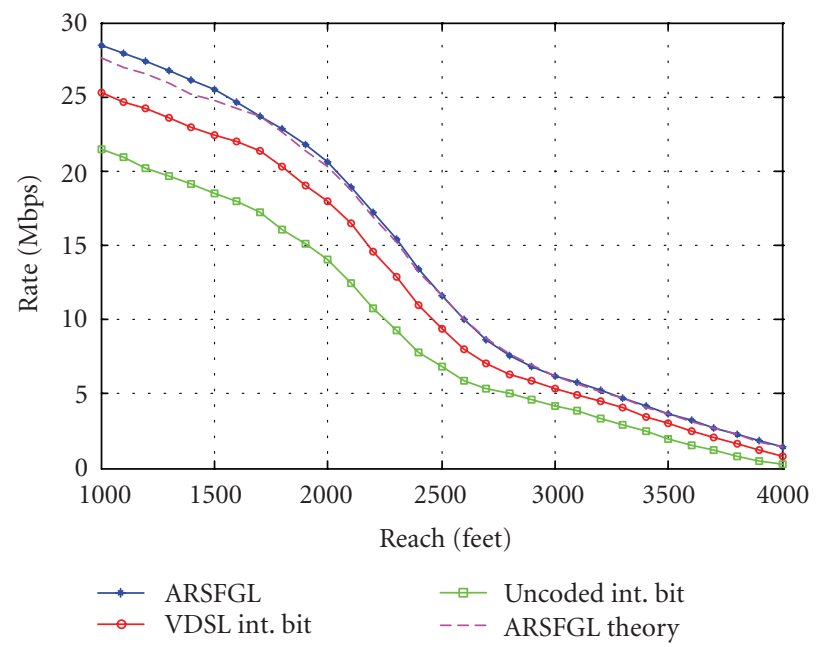

(c)

FIgURE 4: ARSFGL performance for PPO case. (a) Channel signal-to-noise ratio, $\rho$, at $2400 \mathrm{ft}$, (b) sample rate allocation, $b\left(\sigma_{j}\right)$, at $2400 \mathrm{ft}$, and $(c)$ rate versus reach. 


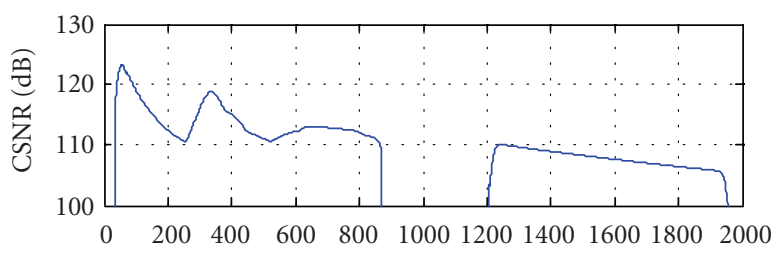

(a)
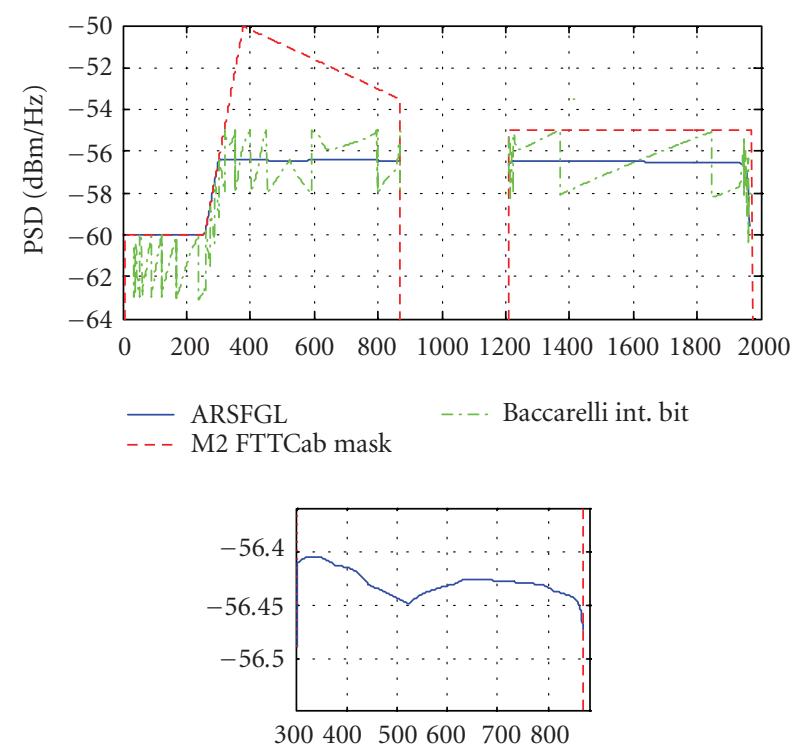

(b)

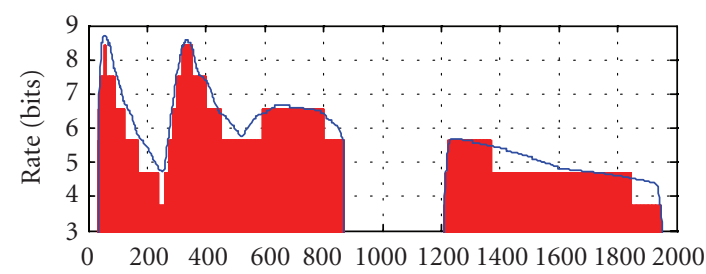

\section{ARSFGL}

Int. bit. (bac.)

(c)

FIGURE 5: ARSFGL performance for TPP case. (a) Channel signal-to-noise ratio, $\rho$, at different subcarriers for $2100 \mathrm{ft}$, (b) transmit PSD $\varepsilon_{j}$ at different subcarriers for $2100 \mathrm{ft}$, and (c) bit distribution $b\left(\rho_{j} \varepsilon_{j}\right)$ at different subcarriers for $2100 \mathrm{ft}$.

algorithm, and the matroid optimal integer-bit algorithm ${ }^{6}$ [11]. For easier comparison of schemes, the percentage improvements over Chow's algorithm have been presented

\footnotetext{
${ }^{6}$ In [11], an optimal solution to the integer-bit TPP problem was proposed. The optimality was proven using the matroid structure of the underlying combinatorial optimization problem. The optimality of the algorithm makes it valuable for benchmarking (in the context of our paper for the ARSFGL scheme), because this is the best any integer-bit scheme (simple or complicated) can do. However, we must also note that the rate achieved by the algorithm in [7], though suboptimal is very close to the optimal rate achieved by [11]. This is observed in Figure 6 for VDSL cases and was also seen in [11, Table 4].
}

in Figure 6(b). From Figure 6(a), we can see that on average, the ARSFGL scheme provides about $2 \mathrm{Mbps}$ improvement over the integer-bit schemes for loops shorter than $5500 \mathrm{ft}$. As expected from (12), for loops longer than $4700 \mathrm{ft}, \eta$ becomes 1 and this case reduces to a PPO case as shown in Figure 6(b), with all the 3 integer-bit schemes giving exactly the same performance. As reach increases, both granularity loss (that depends on $N_{\Omega}$ ) and rate are reduced. However, the reduction in rate is much faster than that in $N_{\Omega}$ (and hence granularity loss). Since the proposed ARSFGL draws most of its improvement from the granularity loss, its percentage of improvement increases with reach as shown in Figure 6(b). 


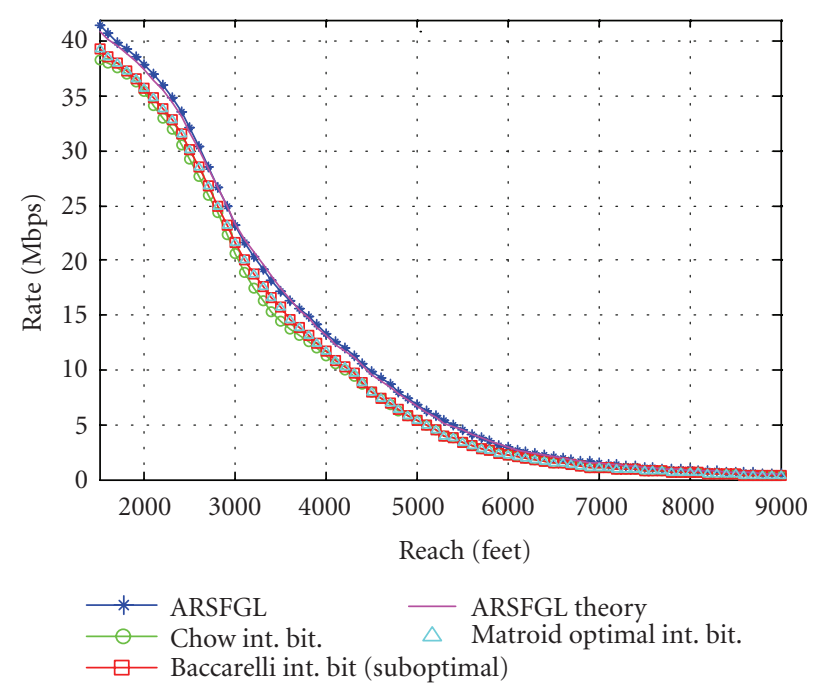

(a)

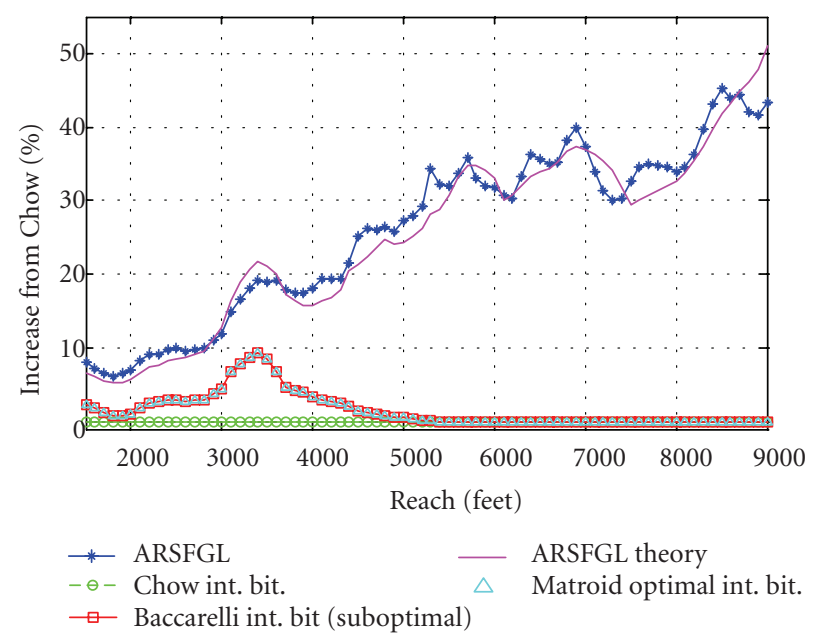

(b)

FIgURE 6: Performance of various schemes for TPP. (a) Rate-reach curves and (b) percent increase in data rate as compared to Chow's algorithm.

The theoretical curves are generated by adding $\overline{\partial b^{G}}$ from (12) to the rate provided by the matroid optimal integer-bit algorithm at different reach values. It is observed that the ratereach curve of the ARSFGL closely follows the theoretical expectations and thereby the assumption on $\eta$ in Section 3 is validated.

\subsection{Evaluation of TPO case}

Though the TPO case does not occur in practice, it has been presented here for the sake of completeness. The hypothetical TPO scenario is constructed by removing the PSD constraint from the TPP configuration shown in Table 3.
The power and rate allocation results of the Leke's algorithm [6] and proposed ARSFGL scheme are shown in Figures 7(a) and 7(b), respectively, for a $2400 \mathrm{ft}$ loop.

Figure 8 shows the percentage increase in rate as compared to Chow's algorithm [5] versus loop lengths offered by the Leke [6], Baccarelli [7], and the optimal (greedy) integerbit Hughes-Hartogs ( $\mathrm{HH}$ ) [9] algorithms and the proposed ARSFGL scheme. It indicates that the rate increase offered by the Leke, Baccarelli, and Hughes-Hartogs algorithms is less than $1 \%$ while the proposed ARSFGL scheme can provide 4-6\% rate improvement for distances up to $7000 \mathrm{ft}$. This improvement is explained by the fact that though in Section 3, we have assumed bit-rounding to be an unbiased operation 


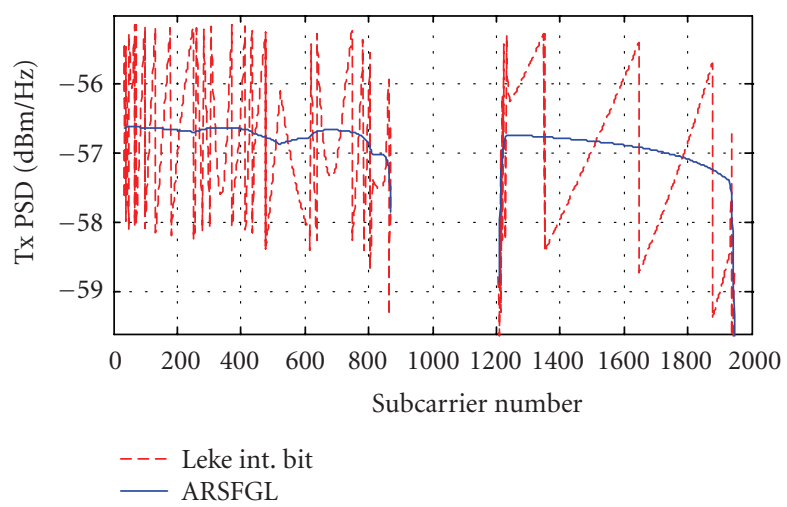

(a)

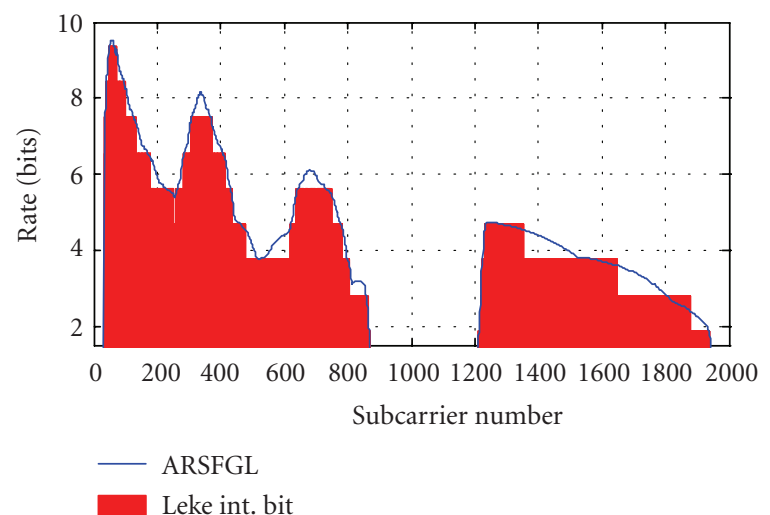

(b)

Figure 7: Power and rate allocation for TPO case and $2400 \mathrm{ft}$ loop. (a) Transmit PSD $\varepsilon_{j}$ at different subcarriers, and (b) bit distribution $b\left(\rho_{j} \varepsilon_{j}\right)$ at different subcarriers.

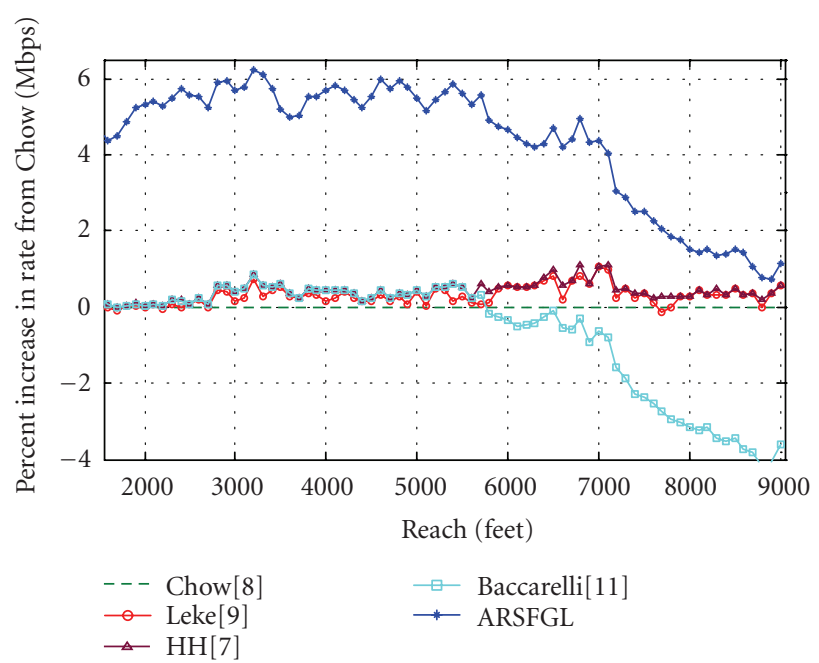

FiguRE 8: Performance of various schemes for TPO case.

for simplifying the analysis, rounding up a bit always costs more in terms of energy than rounding down for the same difference due to the logarithmic (concave) nature of the rate function. This bias leads to the granularity loss being positive even for the TPO case due to $\Omega_{2}$ set of subcarriers. However in PPO and TPP case, as we observed, this effect is strongly dominated by loss due to $\Omega_{1}$.

\subsection{Applicability to dynamic spectrum management}

The above results and analysis have been presented for the case when spectrum management is performed through specification of spectral masks for all users, which is the currently standardized form of spectrum management in ADSL $[2,8]$ and VDSL [3], known as static spectrum management.

Dynamic spectrum management (DSM) techniques have been recently introduced to improve the reach-rate performance of $x D S L$, for example, $[24,25]$. In a DSM case, peakpower constraint still occurs although it is more implicit, and granularity loss still exists. For example, in [26], it was observed that, for a 24-AWG scenario consisting of 4 loops of $600 \mathrm{~m}$ and 4 loops of $1200 \mathrm{~m}$, when the $1200 \mathrm{~m}$ loops are constrained to achieve a minimum of $5 \mathrm{Mbps}$, the $600 \mathrm{~m}$ loops using iterated water-filling (IWF) [27] can achieve 3.4 Mbps and 7.7 Mbps with integer-bit-loading and ideal 
continuous bit-loading, respectively. When optimum spectrum management (OSM) is used in the same scenario, the $600 \mathrm{~m}$ loops achieve $13 \mathrm{Mbps}$ and $15 \mathrm{Mbps}$ with integer-bitloading and ideal continuous bit-loading, respectively. With its near-continuous bit-loading nature, the proposed ARSFGL scheme could be used to recover most of such large granularity losses, that is, to approach the rates offered by ideal continuous bit-loading. Furthermore, given the sawtooth and discrete nature of integer-bit distribution, multiple Nash equilibriums might exist and oscillations around these also seem likely when IWF is used with integer-bit-loading algorithm. This problem could be also mitigated to a large extent by using the ARSFGL scheme.

\section{CONCLUSIONS}

We examined the granularity loss due to the integer-bit restriction that can contribute in a significant percentage in reducing the achievable data rates, especially in peak-power constrained cases, and developed a fine-granularity BERbased loading scheme to recover these losses. This is done by jointly optimizing the coding rate of a programmable $\mathrm{RS}(n, k)$ code and the bit and energy allocation on each subcarrier. Illustrative examples of applications to VDSL-DMT systems indicate that the proposed scheme outperforms various existing integer-bit-loading algorithms with an increase in rate of about $20 \%$ in most cases. This is a large rate increase as compared to the variation in achievable rates of less than $4 \%$ between various existing integer-bit-loading algorithms. This improvement is in good agreement with the theoretical estimates developed to quantify the granularity loss. The theoretical estimates also present an insight into how the granularity losses increase with rising strictness in the peak-power constraint, in comparison to the total-power constraint and with the number of subcarriers in use. Although the illustrative results are for the currently standardized static spectrum management, it is expected that, with its near-continuous bit-loading nature, the proposed scheme can also be used to recover potential granularity losses that exist in dynamic spectrum management (DSM) cases.

\section{ACKNOWLEDGMENTS}

This work is partially supported by a National Sciences and Engineering Research Council Collaborative Research and Development Grant with Laboratoires universitaires Bell. The authors wish to thank Mr. Martino Freda and Mr. Nestor Couras, Electrical and Computer Engineering Department, McGill University, Montreal, Canada, for their indispensable help in channel model construction; and Dr. Ioannis Psaromiligkos, Dr. Jan Bajcsy, and Dr. Harry Leib, all from Electrical and Computer Engineering Department, McGill University, Montreal, Canada, for their insightful discussions. The authors also acknowledge the comments by an anonymous reviewer, who helped to better highlight the improvements of the proposed scheme.

\section{REFERENCES}

[1] J. M. Cioffi, "A Multicarrier Primer," ANSI T1E1.4 Committee Contribution, pp. 91-157, November, 1991.

[2] "Asymmetric Digital Subscriber Line (ADSL) Metallic Interface," ANSI Std. T1.413-1998, 1998.

[3] "Very-high Speed Digital Subscriber Lines (VDSL) Metallic Interface," ANSI Std. T1E1.4/2003-210R5, 2003.

[4] R. G. Gallager, Information Theory and Reliable Communication, John Wiley \& Sons, New York, NY, USA, 1968.

[5] P. S. Chow, "Bandwidth optimized digital transmission techniques for spectrally shaped channels with impulse noise," Ph.D. dissertation, Stanford University, Stanford, Calif, USA, 1993.

[6] A. Leke and J. M. Cioffi, "A maximum rate loading algorithm for discrete multitone modulation systems," in Proceedings of IEEE Global Telecommunications Conference (GLOBECOM '97), vol. 3, pp. 1514-1518, Phoenix, Ariz, USA, November 1997.

[7] E. Baccarelli, A. Fasano, and M. Biagi, "Novel efficient bitloading algorithms for peak-energy-limited ADSL-type multicarrier systems," IEEE Transactions on Signal Processing, vol. 50, no. 5, pp. 1237-1247, 2002.

[8] "Spectrum Management for Loop Transmission Systems," ANSI Std. T1.417-2001, January 2001.

[9] D. Hughes-Hartogs, "Ensemble modem structure for imperfect transmission media," U.S. Patents nos. 4,679,227 (July 1987), 4,731,816 (March 1988) and 4,833,706 (May 1989).

[10] B. S. Krongold, K. Ramchandran, and D. L. Jones, "Computationally efficient optimal power allocation algorithms for multicarrier communication systems," IEEE Transactions on Communications, vol. 48, no. 1, pp. 23-27, 2000.

[11] E. Baccarelli and M. Biagi, "Optimal integer bit-loading for multicarrier ADSL systems subject to spectral-compatibility limits," Signal Processing, vol. 84, no. 4, pp. 729-741, 2004.

[12] J. Jang, K. B. Lee, and Y.-H. Lee, "Transmit power and bit allocations for OFDM systems in a fading channel," in Proceedings of IEEE Global Telecommunications Conference (GLOBECOM '03), vol. 2, pp. 858-862, San Francisco, Calif, USA, December 2003.

[13] P. S. Chow, J. M. Cioffi, and J. A. C. Bingham, "A practical discrete multitone transceiver loading algorithm for data transmission over spectrally shaped channels," IEEE Transactions on Communications, vol. 43, no. 234, pp. 773-775, 1995.

[14] A. V. Oppenheim, R. W. Schafer, and J. R. Buck, Discrete-Time Signal Processing, Prentice-Hall, Englewood Cliffs, NJ, USA, 2nd edition, 1998, (Sec. 4.8.3. Analysis of Quantization Errors).

[15] R. H. Moroles-Zaragoza, The Art of Error Control Coding, John Wiley \& Sons, New York, NY, USA, 2000.

[16] L. Zhang, C. Gao, and Z. Cao, "Exact analysis of bit error rate of maximum-distance-separable codes," in Proceedings of IEEE Global Telecommunications Conference (GLOBECOM '00), vol. 2, pp. 816-819, San Francisco, Calif, USA, November-December 2000.

[17] Telcordia Technologies, "Proposed Bit Rates for Spectral Compatibility with VDSL," ANSI T1E1.4 Committee Contribution, T1E1.4/2002-159R2, August 2002.

[18] M. A. Hasan and V. K. Bhargava, "Architecture for a low complexity rate-adaptive Reed-Solomon encoder," IEEE Transactions on Computers, vol. 44, no. 7, pp. 938-942, 1995. 
[19] Y. R. Shayan and T. Le-Ngoc, "A cellular structure for a versatile Reed-Solomon decoder," IEEE Transactions on Computers, vol. 46, no. 1, pp. 80-85, 1997.

[20] J. G. Smith, "Odd-bit quadrature amplitude-shift keying," IEEE Transactions on Communications, vol. 23, no. 3, pp. 385389, 1975.

[21] K. Cho and D. Yoon, "On the general BER expression of oneand two-dimensional amplitude modulations," IEEE Transactions on Communications, vol. 50, no. 7, pp. 1074-1080, 2002.

[22] "VDSL Test Specification for VDSL Olympics," ANSI T1E1.4 Contribution, T1E1.4/2003-036R4, February 2003.

[23] J. P. Lauer and J. M. Cioffi, "A turbo trellis coded discrete multitone transmission system," in Proceedings of IEEE Global Telecommunications Conference (GLOBECOM '99), vol. 5, pp. 2581-2585, Rio de Janeireo, Brazil, December 1999.

[24] K. J. Kerpez, D. L. Waring, S. Galli, J. Dixon, and P. Madon, "Advanced DSL management," IEEE Communications Magazine, vol. 41, no. 9, pp. 116-123, 2003.

[25] K. B. Song, S. T. Chung, G. Ginis, and J. M. Cioff, "Dynamic spectrum management for next-generation DSL systems," IEEE Communications Magazine, vol. 40, no. 10, pp. 101-109, 2002.

[26] R. Cendrillon, W. Yu, M. Moonen, J. Verlinden, and T. Bostoen, "Optimal multi-user spectrum management for digital subscriber lines," to appear in IEEE Transactions on Communications, http://www.comm.toronto.edu/ weiyu/ osm.pdf.

[27] W. Yu, G. Ginis, and J. M. Cioffi, "Distributed multiuser power control for digital subscriber lines," IEEE Journal on Selected Areas in Communications, vol. 20, no. 5, pp. 1105-1115, 2002.

Saswat Panigrahi received the B.Tech. degree in electrical engineering from the Indian Institute of Technology (IIT), Kanpur, India in 2003. He is currently finishing his M.Eng. degree in electrical engineering at McGill University, Montréal, QC, Canada. His current research interests include multicarrier systems, coding theory, and optimization.

Tho Le-Ngoc obtained his B.Eng. degree (with distinction) in electrical engineering in 1976, his M.Eng. degree in microprocessor applications in 1978 from McGill University, Montreal, and his Ph.D. degree in digital communications in 1983 from the University of Ottawa, Canada. From 1977 to 1982 , he was with Spar Aerospace Limited and was involved in the development
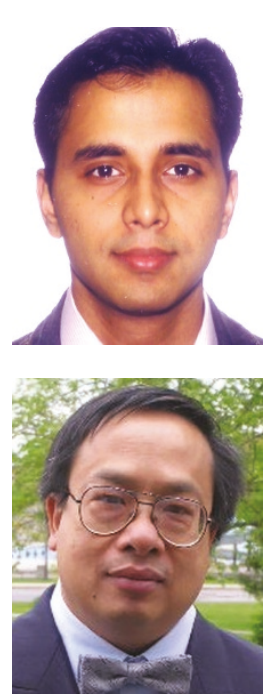
and design of satellite communications systems. From 1982 to 1985, he was an Engineering Manager of the Radio Group in the Department of Development Engineering of SR Telecom Inc. and developed the new point-to-multipoint DATDMA/TDM subscriber radio system SR500. From 1985 to 2000, he was a Professor at the Department of Electrical and Computer Engineering of Concordia University. Since 2000, he has been with the Department of Electrical and Computer Engineering of McGill University. His research interest is in the area of broadband digital communications with a special emphasis on modulation, coding, and multiple-access techniques. He is a Senior Member of the Ordre des ingénieur du Quebec, a Fellow of the Institute of Electrical and Electronics Engineers (IEEE), a Fellow of the Engineering
Institute of Canada (EIC), and a Fellow of the Canadian Academy of Engineering (CAE). He is the recipient of the 2004 Canadian Award in Telecommunications Research and the recipient of the IEEE Canada Fessenden Award 2005. 